\title{
Eradication of fungi from sinuses helps in improvement of lower respiratory tract's clinical symptoms in children with cystic fibrosis
}

This abstract is awarded by ISHAM2018 Attendance grant

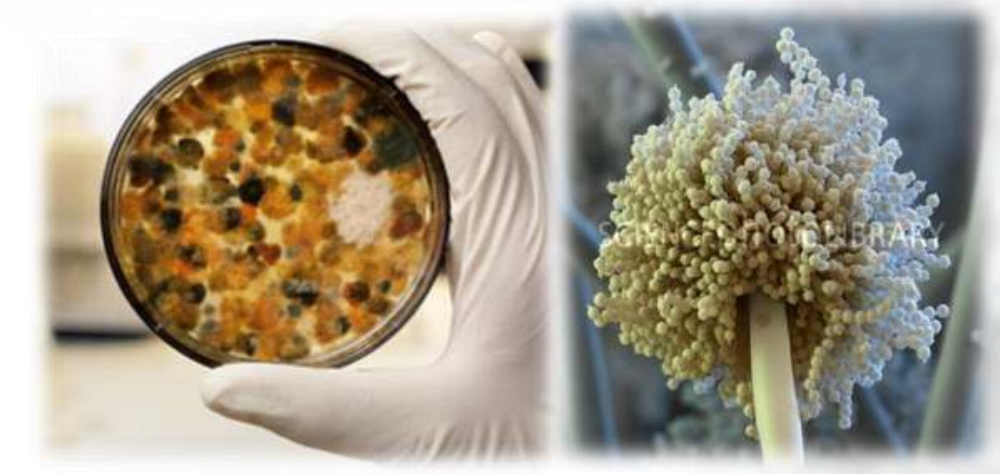

\author{
Aleksandra Barac $^{1,2}$, Predrag Minic ${ }^{2,3}$, Salvatore Rubino 4 \\ ${ }^{1}$ Clinic for Infectious and Tropical diseases, Clinical Centre of Serbia; \\ ${ }^{2}$ Faculty of Medicine University of Belgrade, Belgrade, Serbia \\ ${ }^{3}$ Institute of Mother and Child, Belgrade, Serbia \\ ${ }^{4}$ University of Sassari, Sassari, Italy
}

\section{Introduction}

Although the pathogenesis of cystic fibrosis (CF) and fungal rhinosinusitis (FRS) has been widely investigated, the relationship of these diseases is not revealed yet. The aim of this study was to evaluate the new methodology for detection of fungi in the sinuses of CF patients with FRS, to assess the association of mycobiota of the paranasal sinus and the lower airways of patients with CF and to determine the performance of a functional endoscopic surgery of sinuses (FESS) to clinical symptoms and mycobiota of lower and upper airways.

\section{Results}

Our study revealed: (i) $64.2 \%$ of CF patients had chronic rhinosinusitis (CRS); (ii) fungal presence in sinonasal aspirate was confirmed in $42 \%$ patients (FRS group); (iii) patients with FRS had more severe form of CRS (75.4\%) comparing to nonFRS group ( $p=0.004$ ); (iv) patients with CF and FRS had statistically more complications of CF $(p=0.024)$ and worsen response to therapy $(p=0.03)$ then non-FRS group; $(v)$ all patients from FRS group underwent FESS; $91 \%$ of these patients had improvement of clinical symptoms 3 months after FESS; (vi) fungal presence was confirmed in sputum of $64 \%$ patients with CF, and in $91 \%$ patients from FRS group; (vii) the most commonly isolated species were A. fumigatus (57\%) and A. flavus $(26 \%)$ from sinonasal aspirate and $A$. fumigatus (61\%) and A. alternata (21\%) from sputum (Figure 1); (viii) 3 months after FESS, fungal presence was confirmed in sinonasal aspirate of $7 \%$ and in sputum of $18 \%$ CF patients ( $p=0.009$ and $\mathrm{p}=0.01$; respectively).

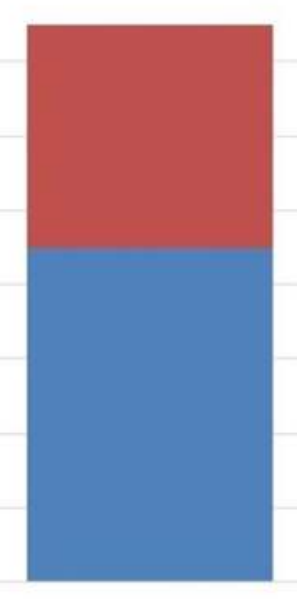

A. fumigatus

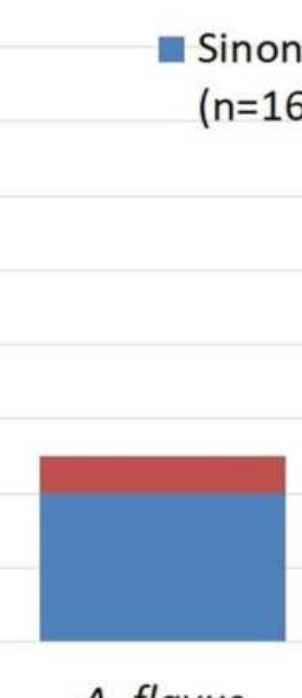

A. flavus

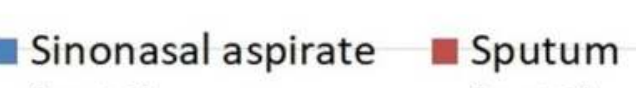

$(n=16)$

$(n=10)$
Figure 1. Fungal strains found in sinonasal aspirate and sputum before FESS in patients from FRS group

\section{Methodology}

The prospective study conducted in 2017 included clinical and mycological data of $38 \mathrm{CF}$ patients. After mycological analyses patients were divided into two groups: (1) patients with confirmed fungal presence in sinuses (FRS group) and (2) patients without fungi in sinuses (non-FRS group). The study design included (Figure 2): 1) anamnestic data; 2) subjective assessment of CRS by sinonasal outcome test; 3) objective assessment of severity of CRS by anterior rhinoscopy; 4) CT imaging of sinuses; 5) mycological examinations of sinonasal aspirate and sputum (before FESS and 3 months after FESS) and 6) functional endoscopic surgery of sinuses (FESS). All clinical and mycological examinations were done before and 3 months after FESS.

\section{Conclusions}

This is the first study that investigates the association of fungal presence in sinuses and sputum of CF patients, and its correlation with clinical findings. There is important correlation between pulmonary and sinus pathogens in CF patients. Subjective and objective clinical symptoms of patients with CF were significantly decreased after FESS. This inseparable connection between fungal diseases of lower and upper airways should be considered when treating these infections in CF patients. A huge amount of fungal spores in the air is the threat for the development of fungal airway diseases and early detection of fungi in the sinuses of these patients could decrease complications of CF and prevent development of invasive fungal diseases.

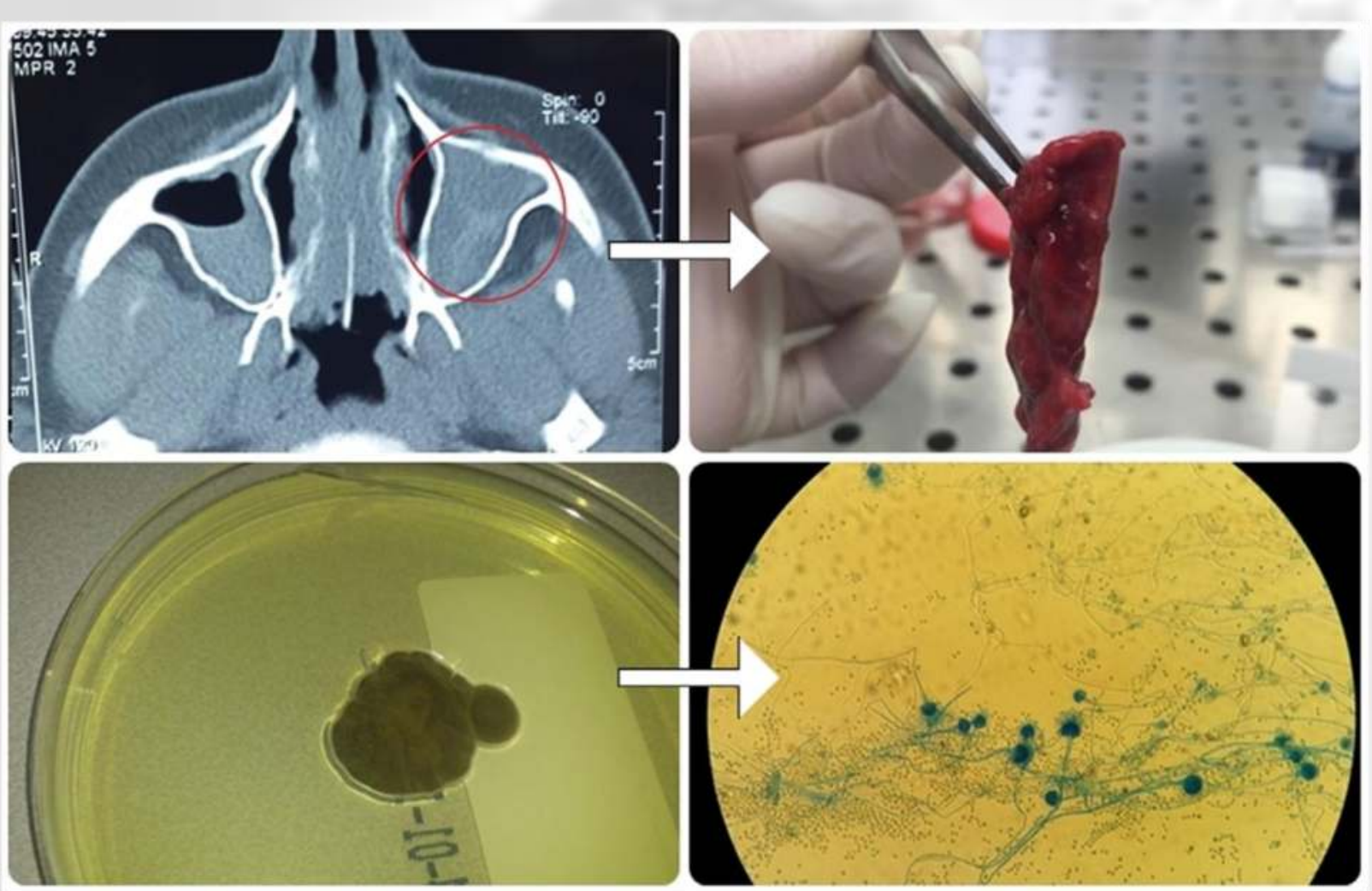

Figure 2. Fungal strains found in sinonasal aspirate and sputum before FESS in patients from FRS group

\section{References}

1. Barac A, et al. Fungi-Induced Upper and Lower Respiratory Tract Allergic Diseases: One Entity. Front Microbiol. 2018 ; 9:583.

2. Agarwal R, et al. Are allergic fungal rhinosinusitis and allergic bronchopulmonary aspergillosis lifelong conditions? Med Mycol. 2017; 55(1):87-95.

3. Ryan MW, et al. Allergic Fungal Rhinosinusitis and the Unified Airway: the Role of Antifungal Therapy in AFRS. Curr Allergy Asthma Rep. 2015; 15(12):75. 